\section{The B Lymphocyte Predominance: Marginal Zone Lymphoma}

\author{
Bajaj A* \\ Histopathologist in A.B. Diagnostics, New \\ Delhi, India
}

* Corresponding author:

Dr. Anubha Bajaj

” anubha.bajaj@gmail.com

Histopathologist in A.B. Diagnostics, New Delhi, India.

Tel: 91-20-26119736

Citation: Bajaj A (2019) The B Lymphocyte Predominance: Marginal Zone Lymphoma. Insights Biomed Vol.4 No.1:3

Keywords: Marginal zone lymphoma; Neoplasm; Sjogren's syndrome

Received: January 02, 2019; Accepted: January 22, 2019; Published: January 25, 2019

\section{Introduction}

\section{Nodal marginal zone lymphoma}

The exceptional neoplasm constitutes an estimated $2 \%$ of the B cell lymphomas with a majority of the subjects presenting with clinical stage III or IV. The indolent lymphoma may demonstrate a clinical course recapitulating follicular lymphoma with divergent clinical manifestations. Nodal marginal zone lymphoma may characteristically be engendered from the lymph node follicles. It may partially recapitulate morphological, immune phenotypic and genetic manifestations of an extra nodal rather than a splenic marginal zone lymphoma [1-3]. Nodal marginal zone lymphoma as an infrequent disorder may account for approximately $10 \%$ of marginal zone lymphomas. The contemporary classification of world health organization (WHO) defines the nodal variant as a primary nodal $\mathrm{B}$ cell neoplasm morphologically identical to a lymph node with attributes of a marginal zone lymphoma of the extra-nodal or splenic subtype in the absence of splenic or extranodal disease. In contrast to splenic and extra-nodal marginal zone lymphoma, the nodal variant depicts an inferior outcome [2].

\section{Clinico-pathological elucidation}

A peripheral lymph node enlargement situated in the head and neck region may be the preliminary presentation. Bone marrow may be implicated in an estimated $30 \%-60 \%$ individuals. Serum chemistries may demonstrate enhanced levels of $\beta 2$ microglobulin and lactate dehydrogenase (LDH) in approximately one third (34\%) of subjects. The serum M component may be detectable in roughly $10 \%$ instances though the percentage may vary. Clinical features, histopathology and disease outcome of the neoplasm may be insufficiently elucidated as nodal marginal zone lymphoma has been described as a separate entity by the world health organization since 2008 [3,4].

Nodal marginal zone lymphoma may be accompanied by autoimmune disorders such as rheumatoid arthritis, vitiligo, systemic lupus erythromatosus, chronic thyroiditis and Sjogren's syndrome with an amplification of infection with hepatitis $C$ virus (HCV). Nodal marginal zone lymphoma may be cogitated as a disorder of obscure pathogenesis [1].

Nodal marginal zone lymphoma may recapitulate follicular lymphoma although the disease may relapse frequently with increasingly aggressive reoccurrences.

\section{Morphological characteristics}

Nodal marginal zone lymphoma may typically depict a parafollicular, peri-follicular and inter-follicular tumour configuration. Columns and cohesive clusters of malignant lymphoid cells may be exhibited which may disseminate or depict distinct tumour 
cell aggregates in the delayed phase, in contrast to the reactive lymphoid follicles. The malignant cells appear to be miniature to medium sized and recapitulate monocytic, centrocytic or lymphocytic cells with a commingling of variable quantities of blastic tumour cells. Nodal marginal zone lymphoma or the alternatively articulated "monocytoid B cell lymphoma" exhibits miniature to medium lymphocyte like cells with spherical or marginally indented nuclei and an abundant, clear cytoplasm. The tumour cells represent the malignant counterpart of monocytoid $B$ lymphocytes discerned in the lymph node sinuses of reactive conditions such as toxoplasmosis [3]. The mode of cellular dispersion may be sinusal and inter-follicular. The transformed monocytoid, centrocytic or lymphocytic cells may colonize and smother the pre-existing lymphoid follicles with the elucidation of floral configurations.

A percentage of malignant cells may cogitate a differentiation recapitulating plasmacytoid cells with the demonstration of intranuclear inclusions reactive to the periodic acid schiff's (PAS) stain termed as "Dutcher's bodies". Nodal marginal zone lymphoma may lack an effortless demarcation from secondary implication of the lymph node with a primary intra-nodal lymphoma or a splenic marginal zone lymphoma. Clinical aspects of specified malignancies may necessitate an extensive delineation $[3,4]$.

Nodal marginal zone lymphoma and lympho-plasmacytic lymphoma (LPL) may depict identical morphological attributes. Lympho-plasmacytic lymphoma may classically be accompanied by immunoglobulin $M$ (IgM) para-protein manifesting as a Waldenstrom macroglobulinaemia. It may be characterized by the MYD88 L265P somatic mutation. Nodal marginal zone lymphoma may also simulate a follicular lymphoma. A reversal of attributes may elucidate a follicular lymphoma replicating a nodal marginal zone lymphoma. Nodal marginal zone lymphoma may typically encompass reactive follicular germinal centers where the tumor cells elucidate an immune reactive $\mathrm{BCL} 2+$ and $\mathrm{MUM} 1+$ with a non-reactive BCL6- and CD10- $[1,3]$. A contemporary marker such as the myeloid cell nuclear differentiation antigen (MNDA) may be a potential tool to demarcate nodal marginal zone lymphoma from a non-reactive follicular lymphoma. A fluorescent in situ hybridization (FISH) analysis may be advantageous in order to discern particular instances with chromosomal translocation $t$ (14:18) (q32: q21) configuring as a specific molecular marker of follicular lymphoma. Distinctive diagnostic and staging methodologies for determining adjunctive marginal zone lymphomas may be mandated and require further elucidation $[4,5]$.

\section{Immune phenotypic markers}

Nodal marginal zone lymphoma may be devoid of a characteristic immune phenotype. Thus, typical immune phenotype or cytogenetic features elucidating nodal marginal zone lymphoma may be challenging to discern. B lymphocytic markers such as CD19+, CD20+, CD79a+ and PAX5+ may be elucidated though nodal marginal zone lymphoma may be non-reactive for CD5, CD10- and CD23-. Concurrent immunoglobulin M (Ig M) and immunoglobulin D (IgD) may be typically exemplified with splenic marginal zone lymphoma, although the feature may be ambiguous and infrequent with nodal marginal zone lymphoma.
Immune markers for plasma cells such as CD38+, CD138+ and MUM1+ may be reactive; implying a prominent plasma cell differentiation [1] (Figures 1-10).

Majority of nodal marginal zone lymphomas may demonstrate frequent somatic mutations of the immunoglobulin heavy chain variable (IGHV) genes, particularly the IGHV3 and IGHV5 subtypes. Trisomy of chromosome 3 and 18 may be enunciated. The diagnosis of nodal marginal zone lymphoma may be one

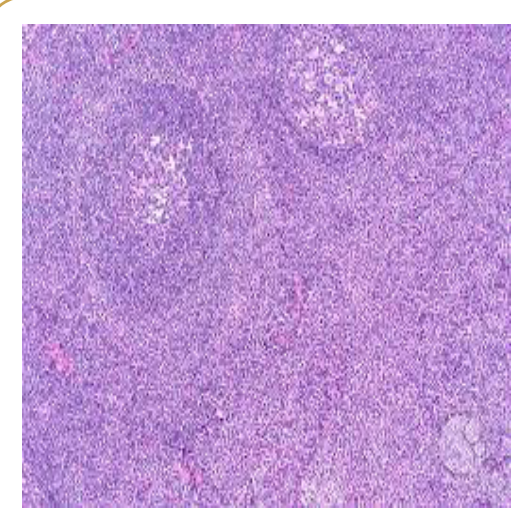

Figure 1 MZL: Monocytoid cells with follicular colonization [17].

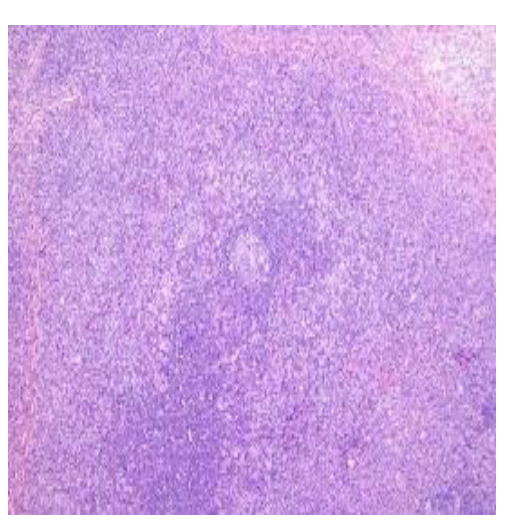

Figure 2 MZL: Malignant lymphoid cells with prominent cellular dispersion [18].

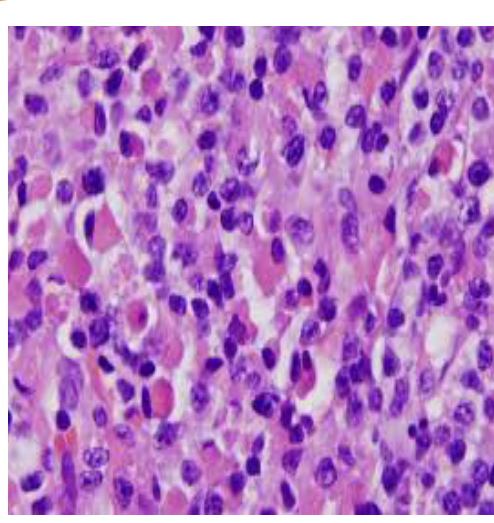

Figure 3 MZL: Plasmacytoid enunciation of nodal marginal zone lymphoma [18]. 


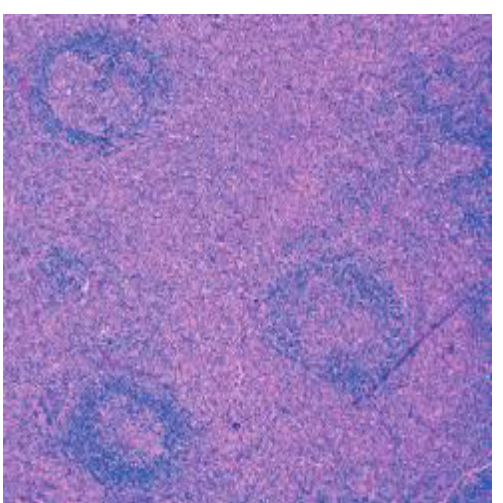

Figure 4 MZL: Inter-follicular configuration of lymphocytic and centrocytic cells [19].

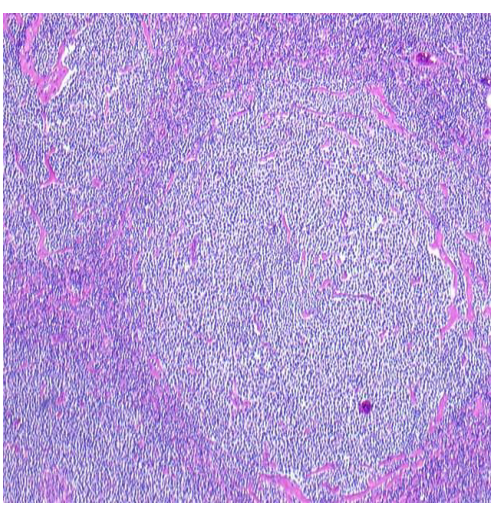

Figure 5 MZL: follicular expansion and colonization of lymphocytic and plasmacytoid cells [20].

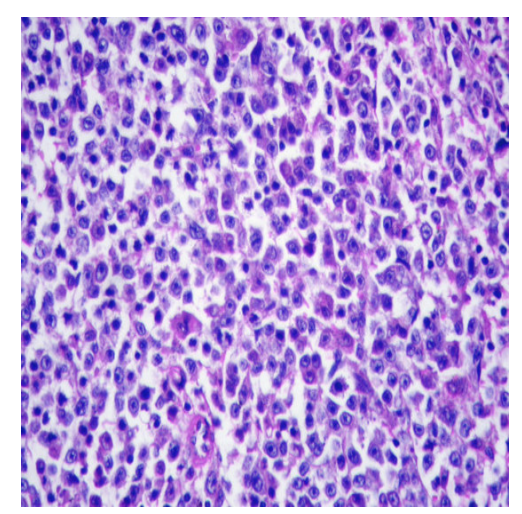

Figure 6 MZL: Plasmacytoid predominance with a sinusal articulation [21].

of exclusion, necessitating a demarcation of the condition from adjunctive diseases with identical attributes. Genetic aberrations elucidated in nodal marginal zone lymphoma as discerned by genomic karyotyping or fluorescent in situ hybridization (FISH) may include chromosomal translocations such as $\mathrm{t}(11: 18), \mathrm{t}$ $(1: 14), t(14: 18)$ and chromosomal deletions such as del $13 q$ and del7q (Figures 11-14) [5,6].

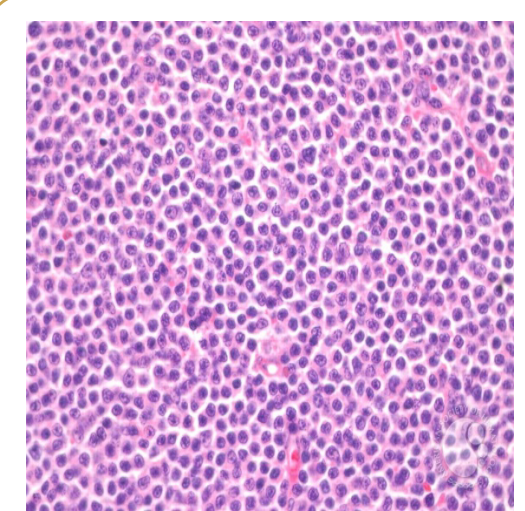

Figure 7 MZL: Inter follicular dispersion of malignant lymphocytic and centrocytic cells [22].

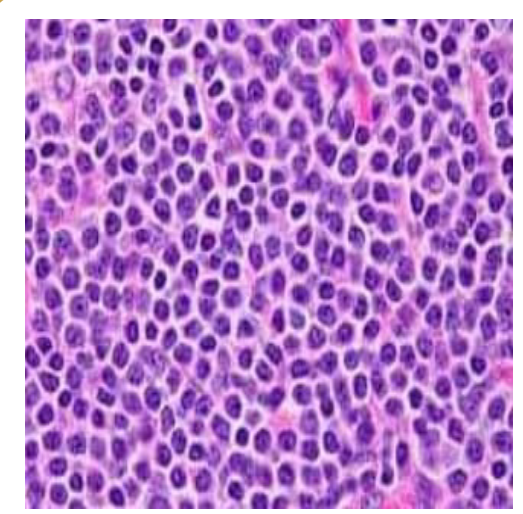

Figure 8 MZL: Admixture of monocytoid and plasmacytoid cells [23].

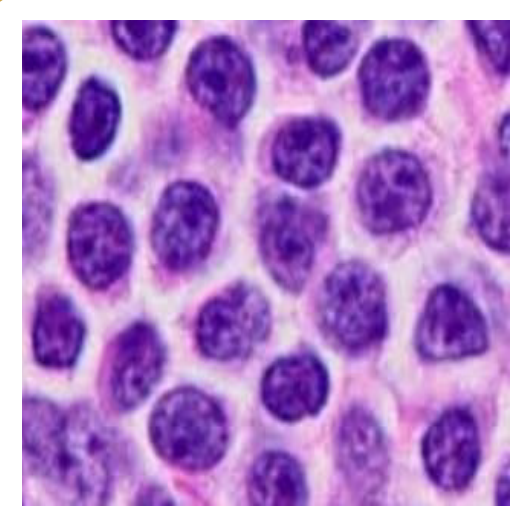

Figure 9 MZL: Monocytoid cells with prominent nucleoli and convoluted nuclei [23].

\section{Molecular aspects}

Comparative genomic hybridization ( $\mathrm{CGH}$ ) of nodal marginal zone lymphoma may recognize reoccurring genomic gains of chromosomes 3,12 and 18 . However, singular genes concurrent with onco-genesis may not be identified. Repetitive and frequent chromosomal translocations of associated lymphoid neoplasm may be lacking in nodal marginal zone lymphoma. A whole exome sequencing (WES) analysis may delineate oncogenic 


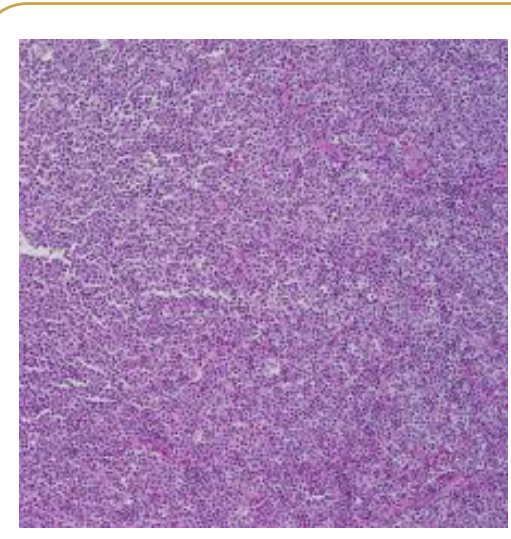

Figure 10 MZL: Sinusal and inter-follicular expanse of centrocytic and monocytoid cells [24].

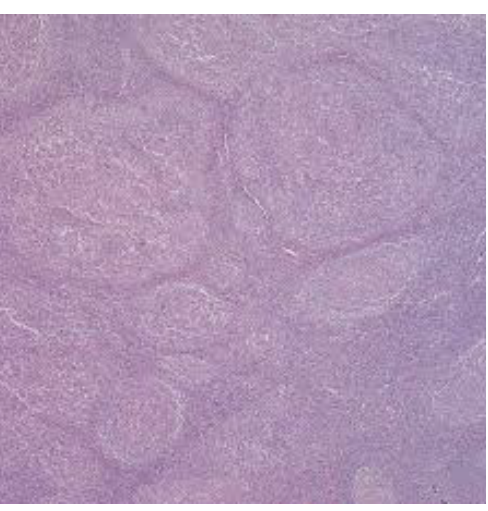

Figure 11 MZL: Follicular colonization of monocytoid and centrocytic tumour cells [25].

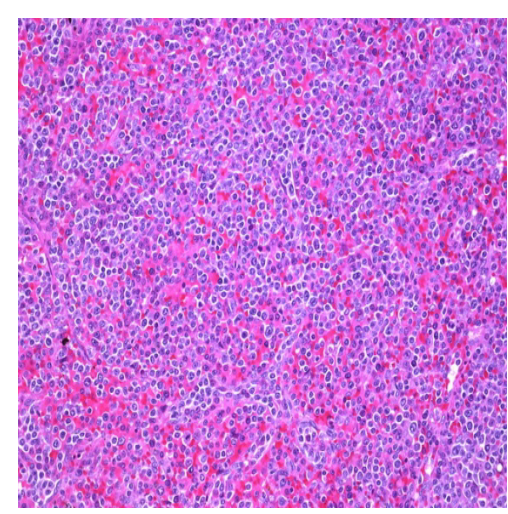

Figure 12 MZL: Spleen with corpuscular aggregation of monocytoid and centrocytic cells [23].

mutations of genes influencing the NOTCH, NFKB, B cell receptor and toll like receptor signaling pathway. Specific mutations may function as genomic biomarkers (PTPRD) or as prognostic biomarkers (KLF2, NOTCH2) for nodal marginal zone lymphoma $[5,6]$. A targeted high throughput sequencing assay may show numerous mutations of the TNFAIP3 gene encoding for A20, the NFKB signaling suppressor. Nodal marginal zone lymphoma mandates a segregation from follicular lymphoma. Thus,

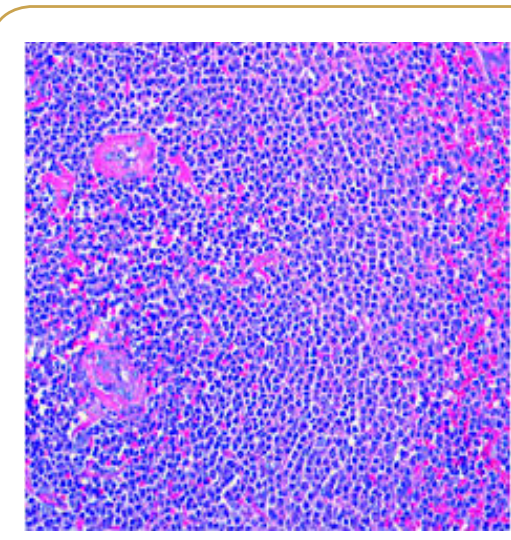

Figure 13 MZL: Spleen with corpuscular aggregation of monocytoid and centrocytic cells [26].

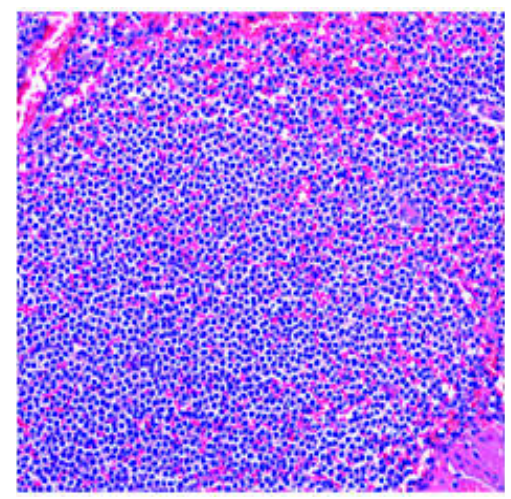

Figure 14 MZL: Spleen with monocytoid and lymphocytoid tumour cells [26].

Table 1 Immune phenotypic and cytogenetic attributes of Nodal marginal zone lymphoma [1].

\begin{tabular}{|c|}
\hline Immune reactive for CD19+ CD20+CD79a+ PAX5+ \\
Immune non-reactive for CD5-, CD10-, CD23- \\
Immune reactive for MNDA. \\
Trisomy of chromosome 3 and 18. \\
\hline Somatic mutations of PTPRD, NOTCH ${ }_{2}, \mathrm{MLL}_{2}$ \\
\hline
\end{tabular}

Table 2 Immune phenotypic and cytogenetic attributes of Splenic marginal zone lymphoma [1].

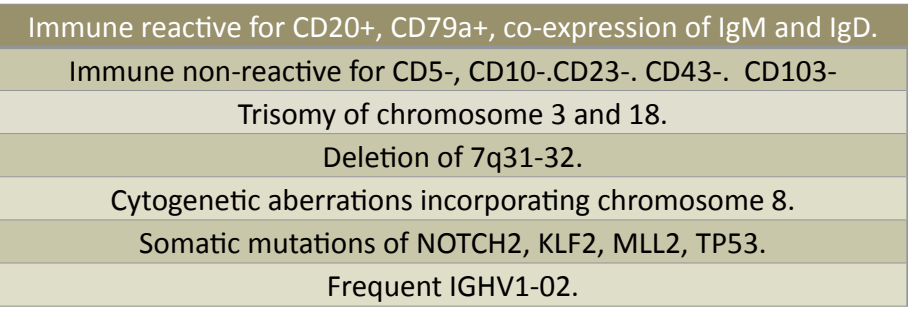

instances of chromosomal translocation $t(14 ; 18)$ with immune reactive $\mathrm{BCL} 2+$ and a mildly reactive $\mathrm{BCL} 6+$ in the absence of a $B C L 6$ genetic rearrangement may be considered as indicative of a nodal marginal zone lymphoma.

Repetitive BRAF mutations may be elucidated in an estimated $16 \%$ of instances along with an intense manifestation of 
Table 3 Differential diagnosis of nodal marginal zone lymphoma [1].

Follicular lymphoma with marginal zone differentiation: BCL-2

negative centroblasts, CD10+ and BCL6+ reactive centrocytes.

Lymphoma is reactive for GCET1, LMO2, HGAL with a t (14:18).

Stathmin1 may be reactive in lymphomas nonreactive to CD10-/

BCL2-

Lymphoplasmacytic lymphoma presents as Waldenstrom

macroglobulinaemia with frequent MYD88L265P mutations.

Cyclin D1/SOX11 immune reactivity may be elucidated in

mantle cell lymphoma.

CD5+ immune reactivity may be enunciated in chronic

lymphatic leukaemia/ small lymphocytic lymphoma.

CD103+ and Annexin A-1 may be exemplified in hairy cell

leukaemia.

Table 4 Evaluation of nodal marginal zone lymphoma [1].

\section{History and physical examination.}

Bone marrow biopsy.

Complete blood counts.

Liver and renal function tests.

Protein electrophoresis.

Serum calcium, lactate dehydrogenase, albumin and $\beta 2$ micro-globulin. Hepatitis $B$ and $C$ virus with human immune deficiency (HIV) virus assay. Helicobacter pylori assay in gastric symptoms.

Computerized tomography (CT) scan of the chest, abdomen and pelvis. Gastro-duodenal endoscopy to exclude extra-nodal disease.

Ear, nose and throat evaluation to exclude extra-nodal disease.

Table 5 Evaluation of splenic marginal zone lymphoma [1]

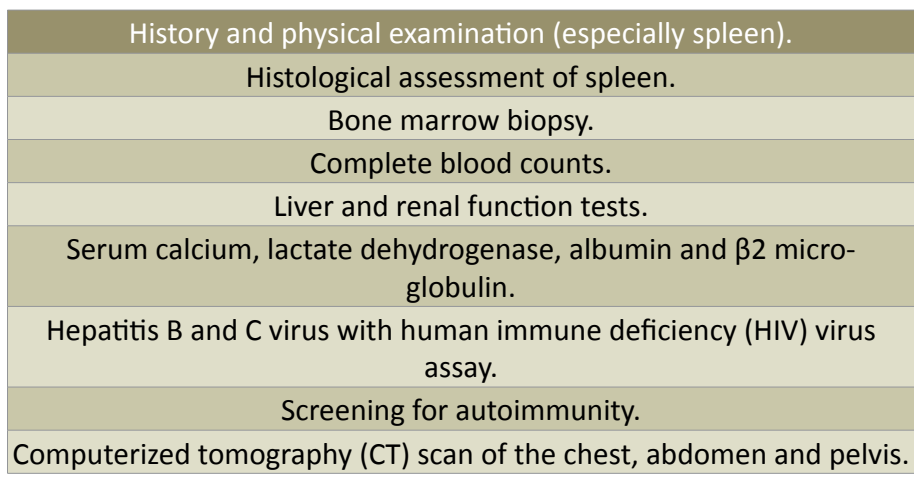

Table 6 Follow up and monitoring of nodal and splenic marginal zone lymphoma [1].

\section{Comprehensive clinical examination.}

Complete blood counts.

Serum chemistries.

For asymptomatic patients every 6 months.

For treated patients at the end of treatment, every 4-6 weeks for first 3 months then every 6 months.

immunoglobulin D (IgD). Additionally, BRAF V600 E mutations may be enunciated in hairy cell leukemia, chronic lymphocytic leukaemia, pro-lymphocytic leukaemia, classic Hodgkin's lymphoma and splenic marginal zone lymphoma. The BRAF molecule encodes for a serine /threonine kinase which may be generally activated by a V600E somatic point mutation. The aforementioned aberration remains a frequent genetic rearrangement in malignant transformation. The particular kinase- activating mutation provides a therapeutic target for BRAF kinase inhibitors [5].

Frequent genetic mutations of nodal marginal zone lymphoma may comprise of TET2, EZH2and BRAF. Associated genomic abnormalities may be mutations of $K M T 2 D$ and recurrent mutations of CREBBP, TNFRSF14, FAS, TNFAIP3, KLF2 and CXCR4 genes. Reoccurring trisomy of chromosome 3, 12 and 18 may be discerned by comparative genomic hybridization (CGH) $[6,7]$.

Nodal marginal zone lymphoma may depict repetitive genetic lesions associated with the development of marginal zone $\mathrm{B}$ cells. Genes such as NOTCH1, SPEN and DTX1 of the NOTCH signalling pathway may be critical for normal differentiation of the marginal zone. Recurrent mutation of the NFKB pathway may be elucidated with nodal marginal zone lymphoma which may also impair genes such as TNFAIP3 and BCL10. Pharmacologic disruption of the NOTCH or NFKB signalling pathway may be appurtenant in nodal and splenic marginal zone lymphoma. Reoccurring mutations in the genes encoding for chromatin remodelling and transcriptional regulation may be discerned. The histone methyl transferase KMT2D, the acetyl transferases CREBBP and TBL1XR1 a critical constituent of the $\mathrm{N}$-Cor co-repressor complex may depict genetic rearrangements [1]. The aforementioned mutations of epigenetic regulators may appear in an estimated $40 \%$ of nodal marginal zone lymphomas and may make therapeutic opportunities with agents such as histone deacetylase (HDAC) inhibitors accessible. Genomic aberrations of BRAF, TET2, EZH2, KMT2D, CREBBP, TNFRSF14 and $F A S$ genes may be found singularly in nodal marginal zone lymphoma. FAS encodes a receptor of the TNF family which may prominently impact extra cellular apoptotic signaling. Genetic mutations, particularly in the protein death domain may eradicate the genomic objective in order to imbue a resistance to the cell for apoptosis. FAS may be frequently mutated in autoimmune lympho-proliferative syndrome, adult $\mathrm{T}$ cell lymphoma and diffuse $B$ cell lymphomas (DLBCL). Appropriate categorization of TNFAIP3, CREBBP, EZH2 and TNFRSF14 may assist in developing an improvised molecular classification of nodal marginal zone lymphoma $[7,8]$.

\section{Investigative assay}

Monocytoid B cells (MBCs) are a subset of B cells that may be recognized in several reactive and tumourous lymph node conditions, including toxoplasma lymphadenitis, infectious mononucleosis and Hodgkin's lymphoma. Although this is a commonly observed cell population, which has even given its name to a type of lymphoma, MBC lymphoma, scarcely any information is available about the function and characteristics of this cell type. Specific markers of $M B C$ are still lacking, and this makes it difficult to analyze their relationship with other $B$ cell subpopulations and to confirm the existence of tumours derived from this cell subpopulation. Reactive lymphocytes predominate in effusions from non-haematopoietic malignancies and benign conditions, while a significant proportion of fluids from patients 
with non-Hodgkin's lymphoma show unequivocal evidence of lymphomatousinvolvement.Immunologicaltyping oflymphocytes in serous effusions is a valuable adjunct to conventional methods of diagnosis particularly in those patients in whom invasive procedures are undesirable. A relationship with marginal zone (MZ) B lymphocytes has been claimed for this B cell subpopulation based mainly on the architectural localization and cytology, but also on the proposed IgM+ or IgD- immune phenotype and the results of mutational analysis of the rearranged immunoglobulin variable genes. Reactive lymphocytes predominate in effusions from non-haematopoietic malignancies and benign conditions, while a significant proportion of fluids from patients of nonHodgkin's lymphoma show unequivocal evidence lymphomatous involvement. Immunological involvement of lymphocytes in serous effusions is a valuable adjunct to conventional methods of diagnosis, particularly in those patients in whom invasive procedures are undesirable. A relationship with marginal zone (MZ) B lymphocytes has been claimed for this B cell subpopulation, based mainly on architectural localization and cytology, but also on the proposed IgM+, IgD- immunophenotype and the results of mutational analysis of rearranged immunoglobulin variable genes. reactive lymphocytes predominate in effusions from non-haemopoietic malignancies and benign conditions, while a significant proportion of fluids from patients with non-Hodgkins lymphoma show unequivocal evidence of lymphomatous involvement. Immunological typing of lymphocytes in serous effusions is a valuable adjunct to conventional methods of diagnosis particularly in those patients in whom invasive procedures are undesirable. Diagnostic manifestations of the nodal variant may recapitulate adjunctive small cell lymphomas such as extra-nodal or splenic marginal zone lymphomas or a lympho-plasmacytic lymphoma Clinical staging of nodal marginal zone lymphoma according to the European Society of Medical Oncology (ESMO) guidelines may recommend analysis such as a complete blood count with serum chemistries as the liver function tests, renal function tests, lactate dehyrogenase (LDH), $\beta 2$ micro-globulin assay and protein electrophoresis [8,9]. Bone marrow biopsy and computerized tomography (CT) scan of the chest and abdomen may be mandated. The employment of a positron emission tomography (PET) scan may not be beneficial in the conventional staging or follow up of nodal marginal zone lymphoma, as per the ESMO guidelines [1].

\section{Therapeutic strategies}

As nodal marginal zone lymphoma is an indolent disorder an active surveillance may be acceptable until constitutional symptoms emerge. The optimal therapeutic applications of nodal marginal zone lymphoma remain undefined though options such as radiotherapy, chemotherapy and/or immune therapy may be available. European Society of Medical Oncology advocates the adoption of treatment modalities applicable for alleviating follicular lymphoma. Localized radiotherapy may be beneficial in appropriately curtailing the tumour in individuals with restricted disease $[9,10]$. A proportion of 24 Gy may be suitable for targeting focal and implicated disease configurations. A minimal quantification ( $4 \mathrm{~Gy}$ ) of radiation therapy may prove to be advantageous for palliative recommendations in stages of dissemination and clinical disease advancement along with the optimal employment of immunotherapy $[10,11]$.
Nodal marginal zone lymphoma may be managed with a combination of rituximab with cyclophosphamide, doxorubicine, vincristine and prednisone (RCHOP) or bendamustine combined with rituximab. Bendamustine with concomitant rituximab may depict identical outcomes as achieved with $\mathrm{R} \mathrm{CHOP,} \mathrm{particularly}$ the progression free survival (PFS), though bendamustinerituximab may depict a reduced toxicity. The initiating therapy may be with antibody drug conjugates such as rituximab, immune modulatory agents such as lenalidomide along with rituximab or the employment of ibrutinib tiuxetan for clinical stage $2 B[11,12]$. Elderly patients may benefit from therapeutic induction with chlorambucil or cyclophosphamide in combination with rituximab or singular application of chlorambucil or cyclophosphamide. Subsequent therapy as a second line option may frequently be the employment of concomitant chemo-immunotherapy or disease alleviation with ibrutumomab, tiuxetan. Ibrutinib may be employed for managing relapsed nodal marginal zone lymphoma. As the nodal marginal zone lymphoma conjectures as an elusive entity, acceptable evidence to engender optimal therapeutic regimens remain undefined $[12,13]$.

\section{Splenic marginal zone lymphoma}

A frequent lymphoma of the spleen may be denominated by the splenic marginal zone lymphoma at an estimated $<2 \%$ of comprehensive lymphoid neoplasm. Median age of disease emergence is roughly 65 years with equivalent gender prevalence. Splenic marginal zone lymphoma represents an indolent, slowly progressive disorder. The median overall survival (OS) may exceed a duration of 10 years, although one third instances delineate an aggressive disease with a consequent decline in the overall survival (OS). In about an estimated $5 \%$ individuals demonstrate a malignant transformation into a "large B cell lymphoma" [1,2].

\section{Discussion}

\section{Clinical elucidation}

Majority of the subjects may be asymptomatic with an accidental discernment of anaemia and thrombocytopenia in the complete blood counts. A clinical emergence of splenomegaly may be associated with anaemia, autoimmune thrombocytopenia and varying quantification of villous lymphocytes in the peripheral blood. With advanced, progressive disease. systemic symptoms may appear on account of splenomegaly such as abdominal discomfort, pain and declining blood counts with pancytopenia $[1,3]$. For appropriate interpretation of splenic marginal zone lymphoma, a correlation of clinical features, laboratory parameters, histological manifestations and radiological aspects may be necessitated, as with associated marginal zone lymphomas.

The etiology of splenic marginal zone lymphoma remains obscure though chronic antigenic stimulation may provide a trigger for the initiation of disease. Splenic marginal zone lymphoma may be frequent with the carriers of hepatitis $\mathrm{C}$ virus (HCV) carriers and eradication of viral infection may induce a remission of the lymphoma. The contingency may be elucidated in southern Italy and regions of North America $[13,14]$. 


\section{Histological elucidation}

Miniature malignant lymphoid cells may constitute the hall mark of the neoplasm. Marginal zone of the white pulp may engender the lymphoma which secondarily invades the hilar lymph nodes of the spleen. The bone marrow infiltrate may elucidate a characteristic sinusoidal configuration. The liver may appear as an adjunctive and frequent site of tumor secondary ingress. Instances of leukemic infiltration and a lack of peripheral lymph node enlargement may be cogitated $[3,4]$.

\section{Immune phenotype}

The malignant lymphoid cells may be immune reactive for $B$ lymphocyte specific cell surface markers such as CD20+, CD79a+ and immunoglobulin M (IgM). Immune molecules such as CD5-, CD10-, CD3- and CD43- may be nonreactive. A demarcation of splenic marginal zone lymphoma may be a pre-requisite from hairy cell leukaemia (HCL). The malignant lymphoid cells of splenic marginal zone lymphoma may be devoid of Annexin (A1) and non-reactive for CD103-. Histological elucidation of the splenic lymphoma may also aid the distinction. Discerning the presence of hepatitis $\mathrm{C}$ virus ( $\mathrm{HCV}$ ) may be crucial as the administration of antiviral therapy may be necessitated advantageous [14,15].

\section{Investigative assay}

A comprehensive medical history with a general physical examination, complete blood counts and serum chemistries such as liver function tests, renal function tests, lactate dehydrogenase (LDH) and $\beta 2$ micro-globulin may be ascertained. An estimated $10 \%-15 \%$ of subjects may demonstrate the emergence of autoimmune diseases as delineated with appropriate evaluation. Attributes computing the prognostic signs may incorporate anaemia, thrombocytopenia, extra hilar lymph node enlargement with an elevated serum lactate dehydrogenase (LDH) and reduced serum albumin values, which may applicable for risk stratification of patients. Tumour invasion extending to non haematopoietic viscera may display an inferior outcome. A splenectomy may no longer be the preferred therapeutic option for managing splenic marginal zone lymphoma as the characteristics diagnostic aspects may be discerned on bone marrow and flow cytometric analysis of the peripheral blood [1-4].

\section{Molecular aspects}

Splenic marginal zone lymphoma may lack the elucidation of specific genetic markers. Trisomy of chromosomes 3 and 18 with a deletion of 7q31.31-q32.3 may be a frequent genetic aberration and characteristic of a splenic marginal zone lymphoma. Somatic mutations of $K L F 2$ gene with anomalies of the NOTCH and NFKB pathway may be concurrent and frequent eventualities. Associated chromosomal aberrations may not be universally discernible. Somatic mutations of the immunoglobulin heavy chain variable (IGHV) gene may be discerned in an estimated half

\section{References}

1 Cogliatti S, Bargetzi M (2016) Diagnosis and treatment of marginal zone lymphoma. Swiss Medical Weekly 146: 1-2.

2 Swerdlow SH (2016) The 2016 revision of World Health
(50\%) of the individuals with concomitant enunciation of IGHV12 and IGHV3-23(1). An estimated one third (34\%) instances of splenic marginal zone lymphoma may present the IGHV1-02 gene and a majority $(90 \%)$ of the subjects may incriminate the allele O4 polymorphic variant (79). The aforementioned findings may implicate an antigenic stimulation and/or a genesis from the progenitor cells sensitive to specific antigenic triggers due to the incitement of particular VH domains $[5,6]$.

\section{Management of Therapeutic strategies}

The management of splenic marginal zone lymphoma may be devoid of specific recommendations. As the neoplasm is indolent, a policy of simple observation may be appropriate. A preliminary therapeutic intervention for patients with mild splenomegaly and a lack of systemic symptoms may not prove to be advantageous. Solitary administration of rituximab or with the addition of ibrutinib may be adopted [14,15] (Tables 1-6).

However, subjects infected by hepatitis $\mathrm{C}$ virus (HCV) may adopt and benefit from an antecedent antiviral therapy. A combination with or singular interferon administration (in the absence of hepatitis $C$ virus infection) may be opted for. Attributes which indicate commencement of therapeutic intervention as per the society of European medical oncology (ESMO) may incorporate i) progressive splenomegaly, ii) progressive decline in the blood cell counts or pancytopenia, iii) declining haemoglobin values of below $<10$ grams/decilitre, iv) platelet count beneath $<80,000$ / $\mu$ Litre and v) an absolute neutrophil count below $<1000$ cells/ $\mu$ Litre [1]. Splenectomy as a conventional therapeutic option may alleviate the splenomegaly with concomitant constitutional symptoms and augment the blood cell counts in a majority. A median progression free survival (PFS) of 5 years may be exemplified. However, surgical extermination of the spleen may not impact the disease incrimination within the bone marrow or peripheral blood. Chemotherapy, immunotherapy or a minimal dose of radiotherapy to the spleen may be employed for subject's ineligible or unwilling for a surgical eradication of the spleen. Chemotherapeutic regimens adopting alkylating agents such as chlorambucil or cyclophosphamide may be administered. Singular agents such as fludarabine may be minimally beneficial. Solitary rituximab or as a combination therapy may procure an enhanced overall survival (OS) and complete response rate (CRR) $[15,16]$.

\section{Conclusion and Follow up}

Asymptomatic patients of splenic or nodal marginal zone lymphoma may be monitored every 6 months. Procedural investigations for treated subjects may be as frequent as in 4-6 weeks during the first three months. An observation for transformation into a high-grade malignant lymphoma may be a pre-requisite for the variants of marginal zone lymphoma.

Organization classification of lymphoid neoplasm. Blood 127: 23752390.

3 Rosai A, Ackerman A (1833) Surgical pathology. https://www.nccn.org/professionals/physician_gls/recently_ updated.aspx 
4 Pillonei V, Juskevicius D (2018) High through put sequencing of nodal marginal zone lymphomas identifies recurrent BRAF mutations. Leukemia 32: 2412-2426.

5 Braci PM, Benavente Y (2014) Medical history, lifestyle, family history and occupational risk factors for marginal zone lymphoma: The inter lymph non-Hodgkin's lymphoma subtypes project. J Natl Cancer Inst Monogr 2014: 52-65.

6 Olszewski AJ, Castillo JJ (2013) Survival of patients with marginal zone lymphoma: Analysis of Surveillance, Epidemiology and End result database. Cancer 119: 629-38.

7 Rinaldi A, Mian M (2011) Genome wide DNA profiling of marginal zone lymphoma identifies subtype specific lesions with an impact on the clinical outcome. Blood 117: 1595-604.

8 Zucca $E$, Bertoni $F$ (2014) Emerging role of infectious etiologies in the pathogenesis of marginal zone lymphoma. Clin Cancer Res 20: 5207-5216.

9 Van den Brand M, Van Krieken JH (2013) Recognizing nodal marginal zone lymphoma: Recent advances and pitfalls; A systematic review. Haematologica 98: 1003-1013.

10 Matutes E (2013) Splenic marginal zone lymphoma: Disease features and management. Expert Rev Haematol 6: 735-745.

11 Montalban C, Abraira V (2012) Risk stratification for splenic marginal zone lymphoma based on haemoglobin concentration, platelet count, high lactate dehydrogenase levels and extra-hilar lymphadenopathy: Development and validation of 593 cases. Br J Haematol 159: 164-171.

12 Ferreri AJ, Govi S (2013) Marginal zone lymphoma and infectious agents. Semin Cancer Biol 23: 431-440.

13 Vannata B, Stathis A (2015) Management of the marginal zone B-cell lymphomas. Cancer Treat Res 2015: 165227-165249.

14 Reid R, Friedberg JW (2013) Management of marginal zone lymphoma. Oncology 27: 840-844.

15 Glehen TA, Bertoni F (2012) Nodal marginal zone B cell lymphoma: A diagnostic and therapeutic dilemma. Oncology 26: 92-94. 